\title{
On the general form of Stevens' law for loudness and softness
}

\author{
R. J. IRWIN AND M. C. CORBALLIS \\ UNIVERSITY OF AUCKLAND, NEW ZEALAND
}

The loudness of a $1000-\mathrm{Hz}$ tone at weak sound pressures and its softness at intense ones were scaled by the method of magnitude estimation. Estimates of loudness plotted as a function of sound pressure on double logarithmic coordinates showed the well-documented steepening near threshold. A similar steepening occurred in the softness function at high intensities. Stevens' law, which is linear on these coordinates, could be recovered either by translating the pressure scale to a new origin, or by translating the response scale. Translations of the response scale were preferred because they produced functions in which loudness and softness were reciprocally related, whereas transformations of the stimulus made the softness function almost three times as steep as the loudness function.

Experimental evidence, contributed principally by S. S. Stevens, has shown that when sensory magnitudes are measured by having subjects make direct estimates of the apparent magnitude of stimuli, then judged magnitude is proportional to physical magnitude, raised to a power. This relation, which we may call Stevens' law, is conveniently plotted as a straight line on double logarithmic coordinates. But a number of dimensions, of which loudness is one, show a steepening of the $\log -\log$ plot near threshold, and therefore a departure from Stevens' law in its simplest form. This paper is concerned with the nature of this departure for loudness and softness.

Two ways have been suggested for transforming functions that exhibit a curvature near threshold so that a linear function can be recovered on logarithmic coordinates. One way, first mentioned by Ekman (1958), is to rescale the stimulus in terms of level above threshold: if a constant magnitude, corresponding to the level of the stimulus at threshold, is subtracted from each stimulus value, then the data again follow Stevens' law over the whole range of stimuli. The other way, which seems to have been first used by McGill (1960), involves rescaling the responses by adding a constant quantity to each judgment. In this way Stevens' law is recovered on the assumption that the subject places the zero of his scale too high.

Published data on loudness near threshold do not allow a clear decision between these two forms of the loudness function. Four papers appeared in 1961 -1962 on this subject, and they divided the two methods equally between them. McGill (1960) showed that loudness functions of individual listeners could be rectified by adding a constant to each listener's estimates.
Galanter and Messick (1961) showed that a translation of either the stimuli or the response rectified their data, but they preferred, on theoretical grounds, to translate the stimli. Scharf and Stevens (1961) achieved the same effect for their data, also by translating the physical scale. Lochner and Burger (1961) asserted that data on loudness published by Hellman and Zwislock (1961) could be rectified only by translating the responses, and this same method rectified some data of their own obtained under various levels of masking.

Fagot (1966) has proposed a theoretical method for discriminating between these two forms of the psychophysical law, but presents no data. A feature of his method is that it requires that the absolute threshold be known by independent measurement. We propose another way of choosing between the two forms of the curve. Our method does not require independent evaluation of threshold. Instead, it examines the consequences of each type of transformation for the attribute that is the opposite of loudness, namely, softness. We present data from subjects who judged the loudness of rather soft stimuli and the softness of rather loud ones. We then examine what transformations make these parts of the loudness and softness functions linear on $\log -\log$ coordinates. Because a number of investigations have shown that, over the major portion of each function, loudness and softness are approximately reciprocally related when both are measured directly (Stevens \& Guirao, 1962, 1963; Eisler, 1962; Schneider \& Lane, 1963), we determine what transformation of both loudness and softness most nearly maintains reciprocity between them. We show that this criterion, and certain others, favor a translation of the sensory rather than the physical scale.

\section{Apparatus}

\section{METHOD}

An Amplivox 82 audiometer generated a $1000 \mathrm{~Hz}$ tone whose level in the S's earphone was controlled by the audiometer's attenuator. The voltage across the earphone, which was calibrated on a 6 cc coupler, was measured with an RMS audio voltmeter (Bruel \& Kjaer, Type 2410), and converted to sound pressure levels with respect to $2 \times 10^{-4} \mathrm{dyne} / \mathrm{cm}^{2}$. The tone was turned on and off manually by the audiometer's silent switch. The duration of each presentation was about 2 sec, although this time was not controlled precisely. 


\section{Subjects}

Ten men, all connected with the Department of Psychology, University of Auckland, volunteered as Ss. Five of them judged the loudness of the $1000 \mathrm{~Hz}$ tone, and five judged its softness. Those Ss who judged loudness were first tested to ensure that all stimuli that they were required to judge were audible. All Ss had had experience at making psychophysical judgments of auditory stimuli.

\section{Procedure}

Each S was instructed to estimate either the loudness of each stimulus or the softness of each stimulus by giving it a number that was proportional to its loudness or softness. A standard stimulus whose loudness or softness was arbitrarily called "10"' was presented first, and the magnitudes of other stimuli were judged with reference to the standard. This procedure is one variant of the method of magnitude estimation (Stevens, 1956). Subjects were given no special practice at estimating loudness or softness. The $S$ sat in a sound-attenuating booth and recorded his judgments on a prepared sheet of paper.

All stimuli were presented monaurally. For those Ss judging loudness, the standard stimulus was approximately $23 \mathrm{~dB}$ sound pressure level. The other stimuli were approximately $8,13,18,28,38$, and 48 dB sound pressure level. For judgments of softness the standard stimulus was approximately $93 \mathrm{~dB}$ sound pressure level, and the other stimuli were approximately $68,78,88,98,103$, and $108 \mathrm{~dB}$ sound pressure level. After the presentation of the standard, all stimuli, including the standard, were presented twice in random order so that each $S$ made two judgments of seven stimuli.

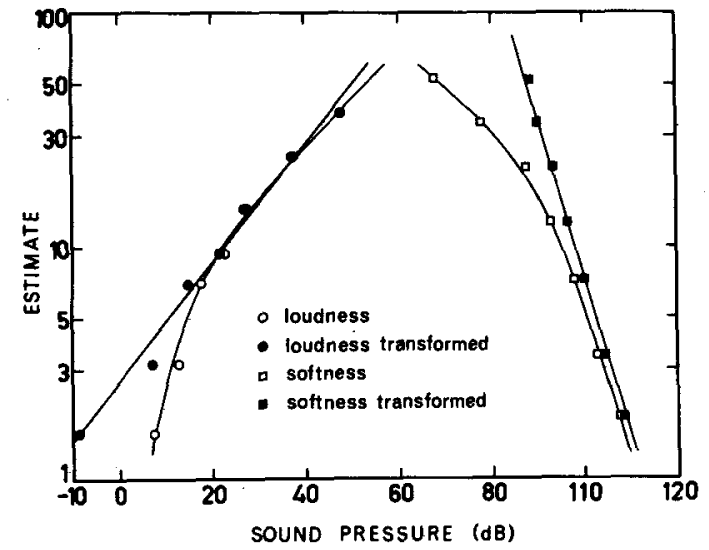

Fig. 1. Estimates of loumess (circles) and softness (squares) as a function of decibel-tevel. Curves have been fitted by eye to the untransformed estimates (open points). Filled points are loudness and softness plotted against a transformed sound pressure scale, $\alpha-\sigma_{0}$, with $\theta_{0}$ chosen to minimize the error of the bestfitting straight line (illustrated).

\section{RESULTS}

The geometric means of the estimates of loudness (open circles) and softness (open squares) are plotted in Fig. 1. All points are based on 10 estimates (two by each of the five Ss), except for the softness of $98 \mathrm{~dB}$, for which one $\mathrm{S}$ withdrew a judgment at the end of the session on the grounds that he had lapsed into a judgment of loudness. This point is therefore based on nine estimates. Other occasional reversals of this sort in the judgment of softness may have occurred. (One potential $S$ had to be rejected because inspection of his estimates of softness revealed that he had unwittingly started judging loudness in the middle of the session.)

The curves drawn through these sets of points have been fitted by eye. The estimates of loudness show the well documented curvature near threshold. Those of softness reveal a similar steepening at high decibel levels. That this curvature, too, may be genuine is suggested by the fact that it has been observed in a number of other investigations of softness, though none of these studies was especially interested in the shape of the softness function at high sound pressures. Curvature can be seen in Eisler's (1962) data on magnitude estimation of a noise band, in Stevens and Guirao's (1962) scale derived from magnitude estimations of a noise band (though not for estimations of a $1000 \mathrm{~Hz}$ tone, nor magnitude productions of either a noise band or a $1000 \mathrm{~Hz}$ tone), and in the same authors (1963) scale of the softness of a noise band based on matches to length of line. Schneider and Lane (1963), however, reported magnitude productions of softness by a single listener in which no curvature occurred.

We next consider transformations of the data in an attempt to recover Stevens' law.

\section{Transformations of the stimulus}

The simplest form of Stevens' law may be written $\psi=k \phi^{n}$ where $\psi$ is sensory magnitude (in our case, loudness or softness), $\phi$ is stimulus magnitude (sound pressure), $k$ is a constant that depends upon units, and $n$ is a constant characteristic of the dimension under study. The law may be generalized to encompass estimates of stimuli near threshold by subtracting a constant quantity, from each stimulus, thus:

$$
\psi=\mathbf{k}\left(\phi-\phi_{0}\right)^{\mathbf{n}} \text {. }
$$

We determined by a method of least squares (see appendix) the value of $\phi_{0}$ that best rectified the judgments of Fig. 1. For loudness this value was $\phi_{0}$ $=6.6 \mathrm{~dB}$. When a constant pressure equivalent to 6.6 $\mathrm{dB}$ was subtracted from each stimulus the data were transformed as shown by the filled circles in Fig. 1. The best fitting line drawn through these points has a slope of 0.509 , which is a little smaller than the generally accepted exponent of 0.54 for monataral loudness. 


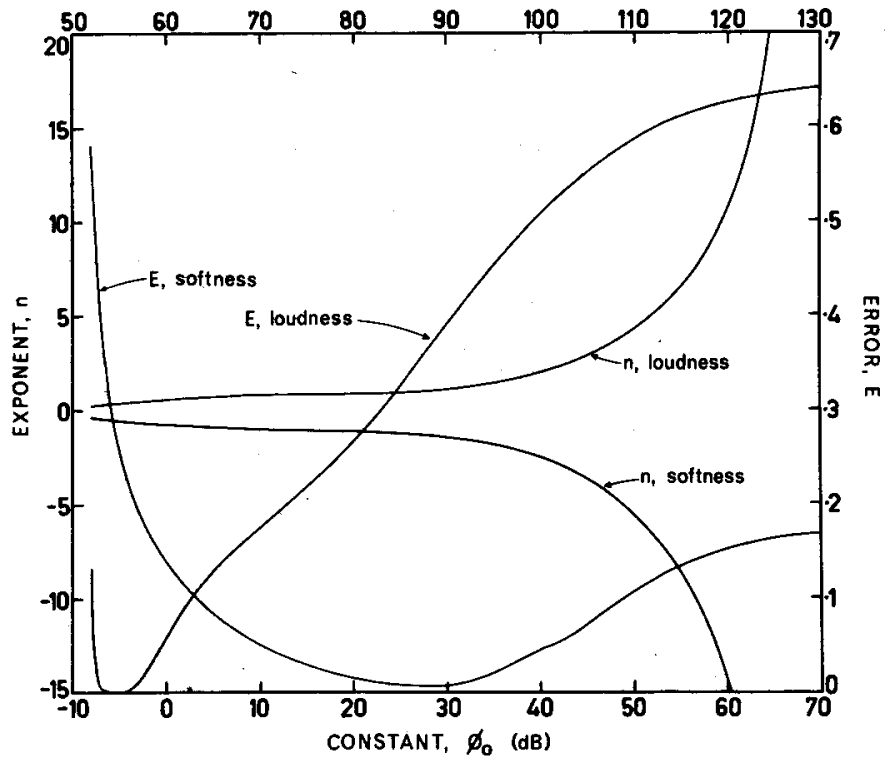

Fig. 2. Best-fitting exponent, $n$, and error, $E$, of the best-fitting line as a function of $\sigma_{0}$ in $\mathrm{dB}$ for the equation $\psi=k\left(\phi-\sigma_{0}\right) n$. The lower abscissa is $\phi_{0}$ for loudness, and the upper is $\phi_{0}$ for softness. The transformations plotted in Fig. 1 are for the case when $E$ is a minimum.

The data for softness also could be rectifled by Equation (1), with the least squares solution for $\phi_{0}$ and $n$ having opposite signs to those for loudness. The best value of $\phi_{0}$ was a pressure equivalent to 87.2 dB which, when added to each stimulus, transformed the softness function in the way shown by the filled squares in Fig. 1. The best fitting straight line has a slope of -1.402 , which is almost three times as large as the exponent of the similarly transformed loudness function.

Figure 2 shows how the best fitting exponent of

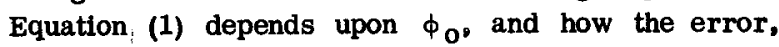
$\mathrm{E}$, in the fit (see appendix) goes through a minimum for both loudness and softness. The transformations depicted in Fig. 1 are for solutions at these minima, and in this sense they are unique solutions.
Equation (1) represents a possible generalization of Stevens' law but it suffers from the twin disadvantages that it generates functions in which loudness and softness are not reciprocally related, and in which the value of $\phi_{0}$ for softness is not readily interpretable. In an effort to overcome this second disadvantage we considered an equation of the form

$$
\psi=k\left(\phi_{0}-\phi\right)^{n}
$$

in which $\phi_{0}$ for softness would have an interpretation analogous to its interpretation for loudness in Equation (1). That is to say; $\phi_{0}$ could be interpreted as the absolute threshold of softness (or the terminal threshold of loudness) which served to rescale the stimuli in terms of level above threshold. An equation of this form was successfully used by Stevens and

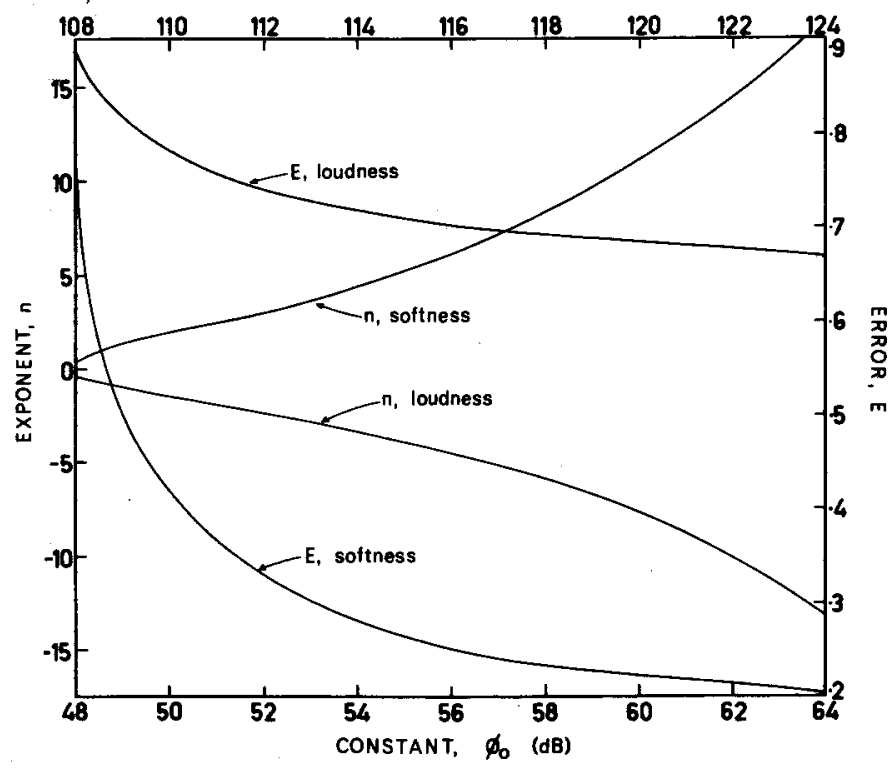

Fig. 3. Best-fitting exponent, $n$, and error $E$, of the best-fitting line as a function of $\phi_{0}$ in $\mathrm{dB}$ for the equation $\psi=k\left(\phi_{0}-\phi\right)^{\mathrm{n}}$. Lower abscissa is $\boldsymbol{\phi}_{0}$ for loudness, and upper is $\phi_{0}$ for softness. $E$ does not pass through a minimum. 


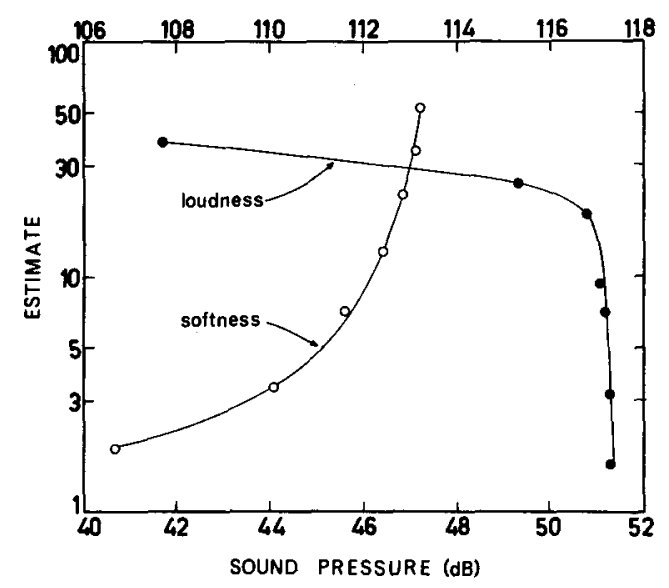

Fig. 4. Estimates of - loudness and softness plotted against a transformed pressure scale, $\phi_{0}-\boldsymbol{\phi}$, for the case where $\partial \mathbf{E} / \partial \boldsymbol{\sigma}_{\mathbf{0}}=$ 0.001. The transformation does not rectify the functions. Lines fitted by eye.

Stevens (1960) to describe the growth of coldness with temperature. The equation failed with our data, however. Figure 3 shows that the residual error, E, does not go through a minimum either for softness or for loudness. There is thus no finite least squares solution to Equation (2). Nevertheless, we considered an arbitrary solution for the case where the first derivative $\partial \mathrm{E} / \partial \phi_{0}=0.001$, and where the exponent of the best fitting line had not reached an excessive value. The result is shown in Fig. 4; the transformation does not rectify either function and so must be rejected.

\section{Transformations of the response}

A generalization of Stevens' law in which responses are translated to a new origin may be written

$$
\psi=\mathbf{k} \phi^{\mathbf{n}}-\psi_{0}
$$

where $\psi_{0}$ is the translating constant. Again, we found the least squares solution for $\psi_{0}$. For loudness this value was $\psi_{0}=14.06$, and the data so transformed are depicted by the filled circles in Fig. 5. (The original estimates are also plotted for convenient comparison.) The best fitting straight line drawn through these points has a slope of 0.268 . The filled squares in Fig. 5 show the transformed data for softness, in which $\psi_{0}=22.33$ yielded the best solution. The slope of the best fitting straight line is $\mathbf{- 0 . 2 6 1}$, which is the same size as the exponent of the comparably transformed loudness function, but opposite in sign. This form of the law therefore leads to the desirable result that loudness and softness are reciprocally related, a finding that is a principal reason for preferring transformations of the response over transformations of the stimulus.

Figure 6 shows that Equation (3) had a unique solution for both loudness and softness, in that each function went through a minimum. The solutions plotted in Fig. 5 are for these minima.
Finally, we also considered translations of the response in which the equation was analogous in form to Equation (2). That is to say, we reversed the signs of $\psi$ and $\psi_{0}$ of Equation (3) to give

$$
\psi=\psi_{0}-k \phi^{n} \text {. }
$$

This function proved unsatisfactory for the same reasons as Equation (2). As Fig. 7 shows, the error functions did not yield a finite least squares solution, and an arbitrary solution for the case where $\partial \mathbf{E} / \partial \psi_{0}$ $=0.001$ did not rectify either function, as can be seen in Fig. 8.

\section{DISCUSSION}

This experiment allows a clear decision on the form of Stevens' law for loudness and softness. It shows that translating the sensory scale by a constant quantity, in accordance with Equation (3), provides the most satisfactory function.

Thus, if the stimuli are translated in accordance with Equation (1), then there are two undesirable consequences: First, loudness and softness are no longer reciprocally related, so that what is twice as loud is not half as soft; and second, $\phi_{0}$ for softness cannot be readily interpreted. But if the stimuli are translated according to Equation (2), then although it is possible to interpret the translating constant for softness as representing the threshold of softness, it turns out that there is no finite value of $\phi_{0}$ that represents a least square solution (Fig. 3), and an arbitrarily good solution does not rectify either function (Fig. 4).

By contrast, if the responses are translated in accordance with Equation (3), then both the loudness and softness functions are rectified, and they remain reciprocally related. In addition, the equation yields a finite least squares solution for both functions. On the other hand, if the responses are translated in

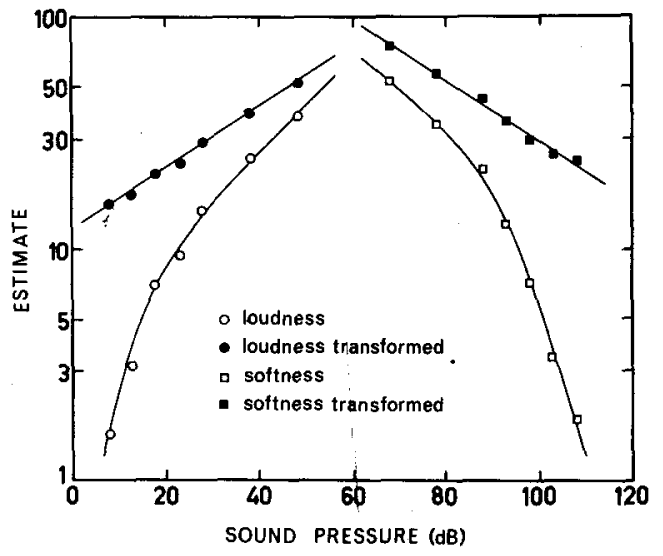

Fig. 5. Filled points are transformed values of loudness and softness, $\psi+\psi_{0}$, with $\psi_{0}$ chosen to minimize the error of the best-fitting straight line (illustrated). The untransformed estimates (open points) are plotted for comparison. 


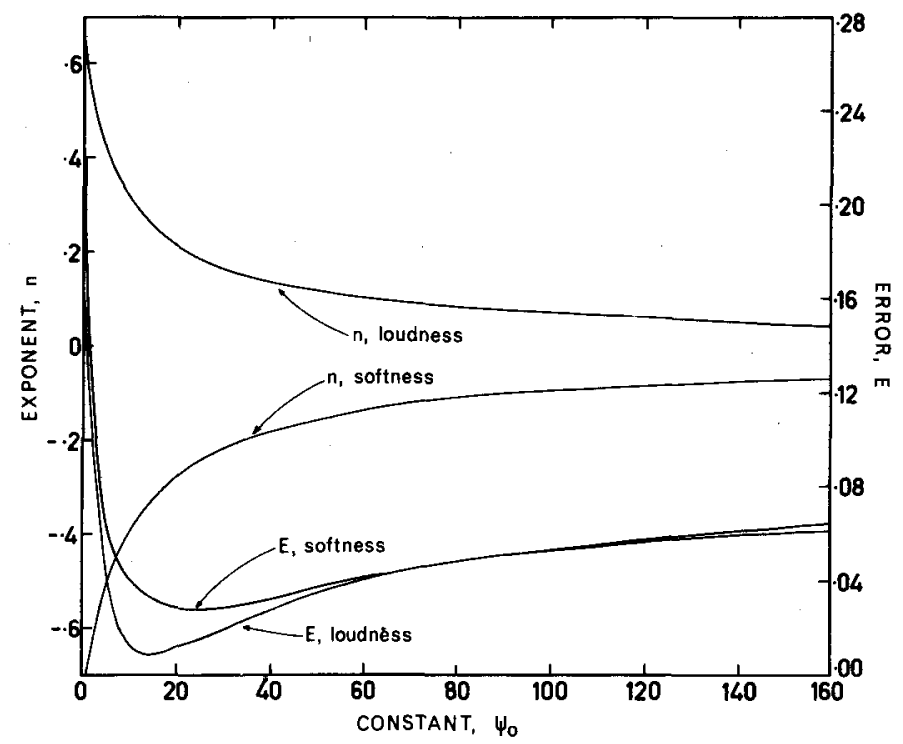

Fig. 6. Best-fitting exponent, $n$, and error, $E$, of the best-fitting line as a function of $\psi_{0}$ for the equation $\psi=$ $\mathbf{k} \boldsymbol{\phi}^{\mathbf{n}}-\psi_{0}$. The transformations depicted in Fig. 5 are for the case when $E$ is a minimum.

accordance with Equation (4), the failings of Equation (2) recur: there is no finite least squares solution (Fig. 7), and an arbitrarily good solution does not rectify the functions.

This experiment shows, therefore, that if the form of Stevens' law for loudness is settled by examining its consequences for softness, then translation of the sensory scale is preferable to translation of the physical scale. Galanter and Messick (1961), however, suggest another consequence that they believe favors a translation of the stimuli. They showed that when loudness is measured by a category procedure, like the method of successive intervals, then it is proportional to the logarithm of direct estimates of loudness. Next they noted that translating direct estimates by an arbitrary constant yields an interval scale, whereas translating stimuli by a unique value, $\phi_{0}$, yields a new ratio scale. They then applied a dictum of Luce (1959), according to which logarithmic relations are not possible between two interval scales, although they are possible between an interval and ratio scale. They therefore concluded that translating the stimuli must be the appropriate form of the loudness function, because translations of the response could not be logarithmically related to category scales, which have interval properties. Even if Luce's dictum is accepted, however, this criterion is inconclusive because the additive constant, $\psi_{0}$, that translates estimates of loudness so that they follow a power function is not arbitrary, but unique. Thus $\psi_{0}$ appears to be no different from $\phi_{0}$ in this respect. For both transformations the only free parameter is $k$, and therefore the scales are ratio ones.

A more cogent reason for preferring to introduce

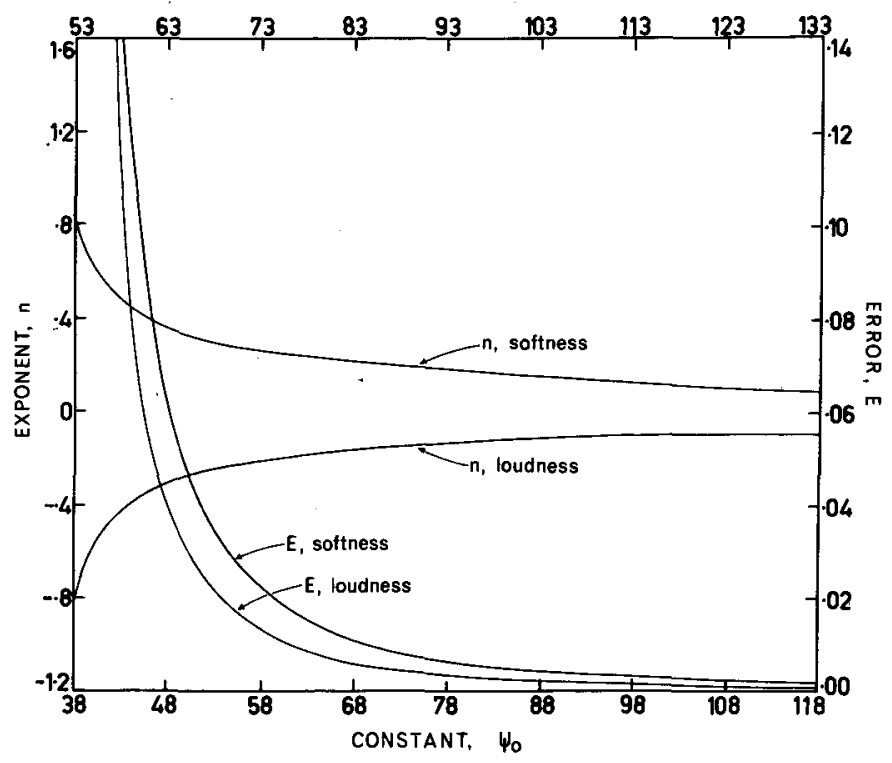

Fig. 7. Best-fitting exponent, $n$, and error, $E$, of the best-fitting line as a function of $\psi_{0}$ for the equation $\psi=$ $\psi_{0}-\mathbf{k} \boldsymbol{\sigma}^{\mathbf{n}}$. $\mathbf{E}$ does not pass through a minimum. 


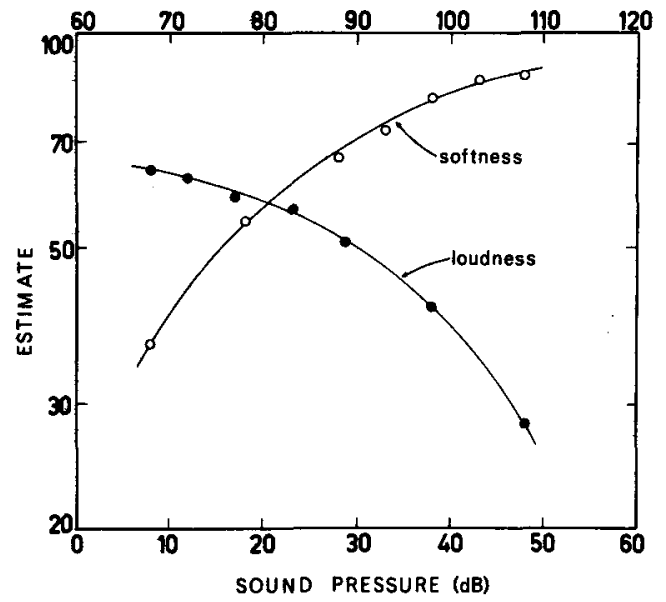

Fig. 8. Transformed values of loudhess and softness, $\psi_{0}-\psi$, plotted against decibel-level for the case where $\partial \mathbf{E} / \partial \psi_{\mathbf{0}}=\mathbf{0 . 0 0 1}$ The transformation does not rectify the functions. Lines fitted by eye.

a threshold parameter in order to rectify the loudness functions is that-for a number of modalities -departures from the simple power function are most readily observable, as Stevens (1959) has noted, whenever the threshold is elevated. These departures have been found for tactile vibration on the arm (Stevens, 1959), for brightness under light adaptation (Stevens \& Stevens, 1963), for warmth and cold (Stevens \& Stevens, 1960), as well as for loudness under masking (Lochner \& Burger, 1961). That a correction for threshold should be most necessary when the threshold differs appreciably from physical zero is intuitively reasonable. Nevertheless, it is possibly not as persuasive as the need to have softness the reciprocal of loudness, nor does it help interpret the nature of the stimulus parameter that rectifies the softness function.

The translating constant, $\psi_{0}$, of Equation (3) implies that the subject places the zero of his scale too high. Why might he do so? Our experiment does not suggest a definitive interpretation, but one possibility is that the subject overreacts to extreme stimulias though, not knowing the limits of his dynamic range, he is too ready to believe that they have been reached. He may therefore assign to stimuli close to his absolute or terminal threshold numbers, or ratios, that are too small, judging the stimuli to be closer to threshold than they really are. Thus $\psi_{0}$ may translate his scale in such a way as to place its origin at the true threshold.

\section{References}

Eisler, H. Empirical test of a model relating magnitude and category scales. Scand. J. Psychol., 1962, 3, 88-96.

Ekman, G. Two generalized ratio scaling methods. J. Psychol., 1958, 45, 287-295.

Fagot, R. F. Alternative power laws for ratio scaling. Psychometrika, 1966, 31, 201-214.

Galanter, E., \& Messick, S. The relation between category and magnitude scales of loudness. Psychol. Rev., 1961, 68, 363-372.
Hellman, Rhona P., \& Zwislocki, J. Some factors affecting the estimation of loudness. J. Acoust. Soc. Amer., 1961, 33, 687-694.

Lochner, J. P. A., \& Burger, J. F. Form of the loudness function in the presence of masking noise. J. Acoust. Soc. Amer., 1961. 33, 1705-1707.

Luce, R. D. On the possible psychophysical laws. Psychol. Rev., $1959,66,81-95$.

McGill, W. J. The slope of the loudness function: a puzzle. In H. Gulliksen \& S. Messick (Eds.), Psychological scaling: theory and applications. New York: Wiley, 1960. Pp. 67-81.

Scharf, B., \& Stevens, J. C. The form of the loudness function neat threshold. In L. Cremer (Ed.), Proceed. 3rd Int. Cong. Acoust. Amsterdam: Elsevier, 1961. Pp. 80-82.

Schneider, B., \& Lane, H. Ratio scales, category scales, and variability in the production of loudness and softness. J. Acoust. Soc. Amer., 1963, 35, 1953-1961.

Stevens, J. C., \& Stevens, S. S. Warmth and cold: dynamics of sensory intensity. $J$. exp. Psychol., 1960, 60, 183-192.

Stevens, J. C., \& Stevens, S. S. Brightness function: effects of adaptation. J. Opt. Soc. Amer., 1963, 53, 375-385.

Stevens, S. S. The direct estimation of sensory magnitudes: Loudness. Amer. J. Psychol., 1956, 69, 1-25.

Stevens, S. S. Tactile vibration: dynamics of sensory intensity. J. exp: Psychol., 1959, 57, 210-218.

Stevens, S. S. Matching functions between loudness and ten other continua. Percept. \& Psychophys., 1966, 1, 5-8.

Stevens, S. S., \& Guirao, Miguelina. Loudness, reciprocality and partition scales. J. Acoust. Soc. Amer., 1962, 34; 1466-1471. Stevens, S. S., \& Guirao, Miguelina. Subjective scale of length and area and the matching of length to loudness and brightness. $J$. exp. Psychol., 1963, 66, 177-186.

(Accepted for publication October 15, 1967.)

\section{APPENDIX}

Finding Best-fit Lines

Equations (1), (2), (3) and (4) can be reduced to the following general logarithmic form:

$$
\log \psi_{1}=\log \mathrm{k}+\mathrm{n} \log \phi_{1}
$$

where $\psi_{1}$ is to be interpreted as $\psi, \psi,\left(\psi+\psi_{0}\right)$ and $\left(\psi_{0}-\psi\right)$ respectively, and $\phi_{1}$ as $\left(\phi-\phi_{0}\right),\left(\phi_{0}-\phi\right), \phi$, and $\phi$ respectively. The equation in $\log \psi_{1}$ and $\log \phi_{1}$ is a straight line. Our problem was to fit such a line to the points $\left(\phi_{1 \mathrm{i}}, \psi_{1 \mathrm{i}}\right), \mathrm{i}=1,7$, where $\phi_{1 \mathrm{i}}$ and $\psi_{1 \mathrm{i}}$ are the data points with translating constants added or subtracted as specified above.

Two ways of fitting the line are, first, to find the form of the equation which predicts $\log \psi_{1 \mathrm{i}}$ from given values of $\log \phi_{1 \mathrm{i}}$, and second, to attempt to predict $\log \phi_{1 \mathrm{i}}$ from values of $\log \psi_{1 \mathrm{i}}$. The former possibility may be expressed by the equation.

$$
\log \psi_{1 \mathrm{i}}^{\prime}=\log \mathrm{k}+\mathrm{n} \log \phi_{1 \mathrm{i}}
$$

where the prime indicates that the $\log \psi_{1 \mathrm{i}}$ are to be predicted from each $\phi_{1 i}$. To fit this equation according to the least squares principle, we must minimize $\mathrm{E}$, where

$$
E=\sum_{i=1}^{7}\left(\log \psi_{1 i}-\log \psi_{1 i}\right)^{2}
$$

Substituting from equation (6) gives

$$
E=\sum_{i=1}^{7}\left(\log \psi_{1 i}-\log k-n \log \phi_{1 i}\right)^{2}
$$


Setting $\partial E / \partial \log k$ and $\partial E / \partial n$ both equal to zero yields two simultaneous equations linear in $\log \mathrm{k}$ and $\mathrm{n}$, which are readily solved to yield values of these constants which minimize $\mathrm{E}$ for any given value of the translating constant ( $\phi_{0}$ or $\psi_{0}$, as the case may be). Our procedure was to solve for $\log \mathrm{k}$ and $\mathrm{n}$ and substitute back into equation (7), for many different values of the translating constant. This was accomplished by computer. $E$ was then plotted as a function of the translating constant.

For equation (1), as is clear from Fig. 2, E for both loudness and softness passes through a minimum for finite values of the translating constant, in this case $\phi_{0}$. These values for loudness and softness were determined to within $10^{-5}$ of their true values by solving the equation $\partial E / \partial \phi_{0}=0$ for $\phi_{0}$, using an iterative procedure. Solutions for $\log k$ and $n$ were obtained for these values of $\phi_{O}$, and the resulting best-fit lines are plotted in Fig. 1.

None of the other equations yielded minimum values for $E$ when the translating constant was finite, or within reasonable bounds. Our next step, therefore, was to reverse the direction of prediction; that is, to find the line, if such exists, which best predicts $\log \phi_{1 \mathrm{i}}$ from given values of $\log \psi_{1 \mathrm{i}}$. This line may be expressed as

$$
\log \phi_{1 \mathrm{i}}^{\prime}=\frac{1}{\mathrm{n}} \log \psi_{1 \mathrm{i}}-\frac{1}{\mathrm{n}} \log \mathrm{k}
$$

We attempted to minimize $E$, now defined as

$$
E=\sum_{i=1}^{7}\left(\log \phi_{1 i}-\log \phi_{1 j}^{\prime}\right)^{2}
$$

or, from (8),

$$
E=\sum_{i=1}^{7}\left(\log \phi_{1 i}-\frac{1}{n} \log \psi_{1 i}+\frac{1}{n} \log k\right)^{2} .
$$

Again, the equations $\partial E / \partial \log k=0$ and $\partial E / \partial n=0$ are readily solved to yield values of $\log \mathrm{k}$ and $\mathrm{n}$ for given values of the translating constant, and substituted back into equation (9). E was plotted against the translating constant. Equation (3) produced minima for finite values of the translating constant $\psi_{\mathrm{o}}$ for both loudness and softness, as is clear from Fig. 6. Solving $\partial E / \partial \psi_{0}=0$ for $\psi_{0}$ and then solving for $\log \mathrm{k}$ and $\mathrm{n}$ for this value of $\psi_{0}$ yielded the best-fit lines plotted in Fig. 5 .

Equations (2) and (4) still failed to reveal minimal values of $E$ for finite values of the translating constant. Consequently, we arbitrarily selected values of the translating constant such that the partial derivative, with respect to the translating constant, of $E$ defined according to equa tion (7), was approximately 0.001 . The resulting transformations plotted in Figs. 4 and 8, however, are clearly unsatisfactory. 